\title{
Adaptive Statistical Iterative Reconstructions Technique (ASIR) Impact on Both Image Quality and Dose in CT Chest Examination
}

\author{
Mariam Isam ${ }^{1{ }^{*}}$, Ayman Mokhtar $^{2}$, A. Abdelrazek ${ }^{1}$, Sabry EL Mogy ${ }^{3}$, A.H. Oraby ${ }^{1}$ \\ ${ }^{1}$ Physics Department, Faculty of Science, Mansoura University, Egypt \\ ${ }^{2}$ Radiology Department, Nephrology and Urology Center, Mansoura University, Egypt \\ ${ }^{3}$ Radiology Department, Faculty of Medicine, Mansoura University, Egypt \\ me.abdelhameed@gmail.com
}

Objective: The main objective of the presented study was to estimate the influence of Adaptive Statistical Iterative Reconstruction (ASIR) algorithm on image and dose Image Quality in Chest CT (Computed tomography) examination compared with the FBP (Filter back projection) techniques.

Methods: Patients were prospectively performed using two scanners at which different reconstruction route were performed: Adaptive Statistical Iterative Reconstruction (ASIR) algorithm in 42 case study, Filtered Back Projection (FBP) in 46 case study, Contrast-to-Noise Ratio (CNR) and Signal-to-Noise Ratio (SNR) were matched between ASIR and FBP images, tested CT images on different percentage ASIR between $0-80 \%$. Then,

ASIR and FBP images were compared, CT dose index volume was documented, Effective doses (EDs) were estimated. Image quality parameters were recorded in the descending thoracic aorta at the level of the carina. Data assessed by two techniques (FBP, ASIR) were compared statistically.

Results: The average image quality in FBP was superior to that of ASIR images. SNR were $(4.03 \pm 1.79$, $3.00 \pm 1.47 \mathrm{BMI}<30)(3.33 \pm 1.33,2.12 \pm 0.94$, BMI >30), CNR were $(2.03 \pm 0.61,1.17 \pm 0.61, \mathrm{BMI}<30),(1.44 \pm 1.01$, $1.11 \pm 0.53, \mathrm{BMI}>30$ ) for FBP, ASIR respectively. A noted significant increase in both CNR and SNR with an increased percentage of ASIR. ASIR had a statistically significantly $(P=0.003)$ lower CTDI $_{\mathrm{vol}}(9.02 \pm 2.01)$ than the conventional FBP $(13.52 \pm 4.01)$, with the use of ASIR, ED was slight differ compared with FBP, the ED values were $\left(7.46 \pm 2.45\right.$, to $9.02 \pm 2.01$, BMI $\left.<30 \mathrm{~kg} / \mathrm{m}^{2}\right),(6.63 \pm 1.59$, to $8.22 \pm 5.13$ with $\mathrm{BMI} \geq 30 \mathrm{~kg} /$ $\mathrm{m}^{2}$ ) for FBP, ASIR respectively.

Conclusion: ASIR enables improving image quality and has the potential to decrease radiation dose in comparison with conventional FBP reconstruction route. It was concluded that clinical assessment is needed to settle the decreasing potential of radiation dose with ASIR

Keywords; ASIR; FBP; CNR; SNR; BMI; DLP

Language: English

Date of Publication: 31-07-2018

DOI: 10.24297/jap.v14i2.7510

ISSN: 2347-3487

Volume: 14 Issue: 2

Journal: Journal of Advances in Physics

Website: https://cirworld.com

This work is licensed under a Creative Commons Attribution 4.0 International License. 


\section{Introduction}

Computed tomographic (CT) route has much superior diagnostic accuracy in different clinical circumstances in comparison to other chest radiography. Although argumentative, contemporary data acquiesce that medical radiation exposure increases significantly the risk effects of contrary radiation [1, 2]. The cancer risk era based on current $C T$ usage has been appointed to be as high as $2.0 \%$ as reported by different authors [3]. Accumulative risks may be higher for certain adult patient populations, particularly for those requiring to follow up studies, addition in obese patients asking increased radiation dose for sufficient tissue penetration.

A number of $C T$ routines sophisticated to reduce radiation dose, however, preserving image quality. Nowadays used regimes include but not limited to reduction of tube voltage [4] combined with automatic modulation of tube current [5], using high pitch, among others [6], and decreased scan length [7]. The standard CT reconstruction algorithm FBP cannot produce diagnostic quality images combined with a reduction of $x$-ray tube currents expressed in $(m A)$. Because inherent in lowering the $\mathrm{CT}$ radiation may lead to an increase in the image noise.

Recently Campell et al [8] introduced a method used to reduce noise depending on the algorithm of iterative reconstruction using complex method for reconstructing images in addition to the ASIR route which has a superior dose reduction combined with preservation and enhancement of the diagnostic proficiency of CT examination [8].

Based on raw pixels ASIR may be considered as a superior CT reconstruction algorithm that use information attained from FBP algorithm as a first step for the process of image reconstruction followed by using matrix algebra (ASIR route) for the transformation of measured value of specific pixel to a new estimated pixel value that compared with the original ideal value predicted. The whole process is recurrent iteratively with small successive steps until a final ideal estimated pixel value converge and identified for image noise reduction [9].

The main goal of the presented study is for estimation of ASIR algorithm on the image and dose quality in $\mathrm{CT}$ Chest examination in comparison with FBP routine.

\section{Materials and Methods}

\subsection{Patient classification}

Presented study includes the CT chest examination of 88 patients in the period of June 7, 2017, and January 20,2018. Selected patients distributed randomly within the study period of prospective annexation. CT chest examinations were operated at (MANSOURA ADVANCED RADIOLOGY CENTER) Mansoura city, Egypt.

The patients were divided into two main groups (Group X) and (Group Y). Group X: 46 patients have been scanned on the CT scanner (BrightSpeed, GE Healthcare 8 detectors-USA) and reconstructed using the FBP technique (the age range of Group $X$ lie between 20 and 79 years with mean age value of about 47.82 years. The study included 18 men and 28 women). Group $Y$ include 42 patients subjected to CT scanner (Revolution EVO, GE Healthcare 128 detectors, USA ) and reconstructed using ASIR technique(10\% to $100 \%$ ASIR in 10\% increments) (the age range of Group Y lie between 30 and 85 years with age mean value of about 50.67 years. The study included 22 men and 20 women). All patients under investigation were scanned without contrast. The demographic data of patients were serene from booking demand forms while their body mass index (BMI) was calculated and introduced in (Table 1). 
Table 1. Patient CT demographic data

\begin{tabular}{|c|c|c|c|c|c|c|}
\hline \multirow{3}{*}{ Characteristic } & \multirow{2}{*}{\multicolumn{2}{|c|}{$\begin{array}{c}\text { FBP } \\
(n=46)\end{array}$}} & \multirow{2}{*}{\multicolumn{2}{|c|}{$\begin{array}{c}\text { ASIR } \\
(n=42)\end{array}$}} & \multirow{3}{*}{$\begin{array}{c}\text { Test of } \\
\text { significance }\end{array}$} & \multirow{3}{*}{$\begin{array}{c}\text { p- } \\
\text { value }\end{array}$} \\
\hline & & & & & & \\
\hline & No & $\%$ & No & $\%$ & & \\
\hline \multicolumn{7}{|l|}{ Gender } \\
\hline Male & 18 & 39.1 & 22 & 52.4 & $\chi^{2}=0.777$ & 0.378 \\
\hline Female & 28 & 60.9 & 20 & 47.6 & & \\
\hline \multicolumn{7}{|l|}{ Age/years } \\
\hline Mean $\pm S D$ & \multicolumn{2}{|c|}{$47.82 \pm 16.55$} & \multicolumn{2}{|c|}{$50.67 \pm 14.94$} & $t=0.595$ & 0.555 \\
\hline Min-Max & \multicolumn{2}{|c|}{$20-79$} & \multicolumn{2}{|c|}{$30-85$} & & \\
\hline \multicolumn{7}{|l|}{ BMI pre } \\
\hline Mean \pm SD & \multicolumn{2}{|c|}{$31.60 \pm 7.06$} & \multicolumn{2}{|c|}{$31.81 \pm 6.84$} & $t=0.097$ & 0.923 \\
\hline Min-Max & \multicolumn{2}{|c|}{$22.04-52.08$} & \multicolumn{2}{|c|}{$24.32-49.54$} & & \\
\hline
\end{tabular}

\subsection{Protocol}

The scanning range for patients on both scanners was from supraclavicular space to the upper abdomen, together with bilateral adrenals gland. The two CT scanners parameters protocols are shown in Table 2.

Table 2: Scanning protocols for Brightspeed (FBP) and Revolution EVO (ASIR) techniques:

\begin{tabular}{ccc}
\hline $\begin{array}{c}\text { Scanning } \\
\text { parameter }\end{array}$ & Brightspeed(FBP) & $\begin{array}{c}\text { Revolution EVO } \\
\text { (ASIR) }\end{array}$ \\
\hline Scan type & Helical(Spiral) & Helical \\
Rotation time & $0.8 \mathrm{~s}$ & $0.6 \mathrm{~s}$ \\
Detector row & 8 & 128 \\
Slice thickness & $2.5 \mathrm{~mm}$ & $2.5 \mathrm{~mm}$ \\
Beam collimation & $20 \mathrm{~mm}$ & $91.66 \mathrm{~mm}$ \\
Pitch & $1.35: 1$ & $1.373 / 1$ \\
Speed & $27 \mathrm{~mm} / \mathrm{rot}$ & $55 \mathrm{~mm} / \mathrm{rot}$ \\
S FOV & large $50 \mathrm{~cm}$ & Large $50 \mathrm{~cm}$ \\
KVP & 120 & 120 \\
Auto mA & $150-220$ & $100-500$
\end{tabular}


recon 1

recon 2
Standard

Lung standard

Lung

The scanner automatically selects ASIR noise reduction level that spans between 10:100\% with $10 \%$ step. Such levels essentially infer level of blending of both FBP and ASIR regime to obtain variable noise reduction of the reconstructed images. Additionally, operated scanner may reconstruct both FBP and ASIR image based on specific level of used ASIR until final reconstructed image data sets were obtained. For example, blended reconstructed image data set will have about $70 \% \mathrm{FBP}$ and $30 \%$ ASIR if the noise reduction level for ASIR was selected to be $30 \%$ while $80 \%$ noise reduction level shows $80 \%$ ASIR and $20 \%$ FBP blending notable low image noise. Although higher noise reduction levels provide lower noise images. In such cases, the vendor recommends the use of $30 \%$ to $50 \%$ ASIR generally.

\subsection{Image Quality}

The subjective and objective image quality calculations of selected groups including $88 \mathrm{CT}$ chest examinations were achieved using diagnostic workstation (Picture Archiving and Communication System) (PACS) using circular region of area $2.0 \mathrm{~cm}^{2}$ centered at descending thoracic aorta mediastinal images. Standard Deviation (SD) in a combination of mean value for the required region of interest was documented noting that SD can be considered as noise and mean can be interpreted as signal.

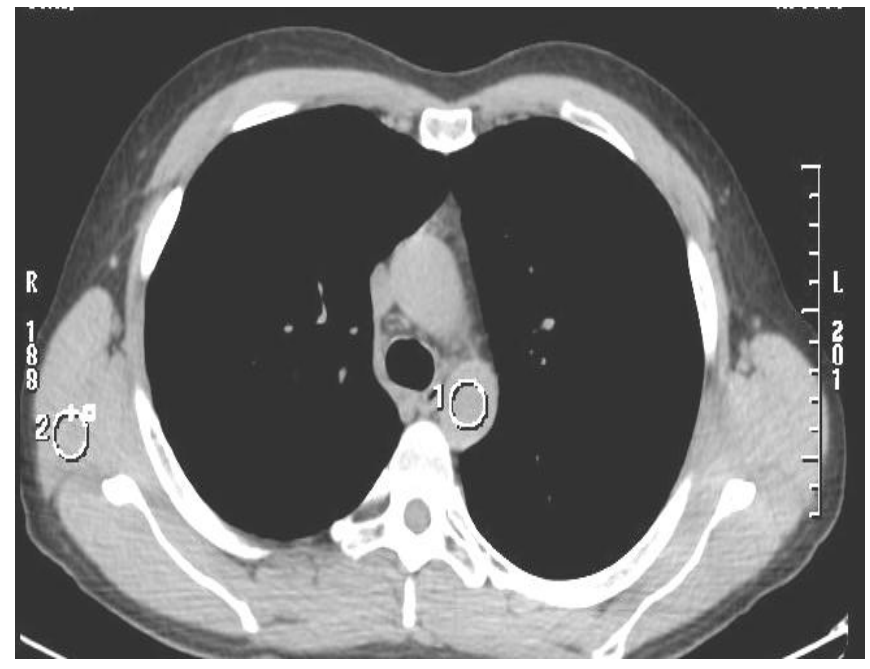

Figure 1. circular region of area $2.0 \mathrm{~cm}^{2}$ centered at descending thoracic aorta mediastinal images.

The method described elsewhere [10] Szucs-Farkas et al. was employed to calculate both contrast-to-noise ratio (CNR) and signal-to-noise ratio (SNR), using equation 1 and 2.

$$
\begin{aligned}
& S N R=\frac{S I_{\text {vessel }}}{\text { Noise }} \\
& C N R=\frac{S I_{\text {vessel }}-S I_{\text {muscle }}}{\text { Noise }}
\end{aligned}
$$

where

$S I_{\text {vessel }}$ represent the intensity of mean signal for pulmonary 
$S I_{\text {muscle }}$ represent the difference between attenuation of protocol center and deep paraspinal muscles and

Noise is defined as mean of standard deviation of recorded measurements.

\subsection{Radiation Dose}

To estimate dose parameters for the 88 chest $C T$ examination results reconstructed using FBP or ASIR technique such as CT dose index volume (CTDI vol ) and dose length product (DLP) were recorded for every patient from the dose report. The effective dose in millisieverts (EDs) was calculated by multiplying dose-length product $\times$ thoracic conversion $\mathrm{K}$ factor of $0.017 \mathrm{mSv} \mathrm{mGy}^{-1} \mathrm{~cm}^{-}$ as described in the EUR16262 document [11]

\subsection{Statistical analysis}

Both of the two patients groups $X$ and $Y$ were also divided into two subgroups based on the Body Mass Index (BMI) (less than $30 \mathrm{~kg} / \mathrm{m}^{2}$, and $30 \mathrm{~kg} / \mathrm{m}^{2}$ or more). Data were analyzed with standard statistical software (SPSS version 21. The normality of data was first tested with Shapiro test. The paired Student's t-test was used to compare two techniques, image quality (signal-to-noise ratio (SNR), contrast-to-noise ratio $(C N R))$ and doses $\left(C^{2} D_{\text {vol }}\right.$, DLP, EDs). It was of interest to determine if image quality, CNR, SNR, a proportion of interpretable segments, or study quality by the Likert scale differed (LSD) by percentage of ASIR used in the reconstruction $(0 \%, 30 \%, 50 \%, 80 \%$,). In addition, Qualitative data were described using number and percent. Association between categorical variables was tested using Chi-square test. Continuous variables were presented as mean \pm SD (standard deviation). Repeated measured ANOVA was used to compare means in different doses. Pearson correlation was used to correlate continuous data The smaller the $p$-value obtained, the more significant are the results, $p<0.05$ was considered statistically significant.

\section{Results}

Imaging was performed on 88 patients; ASIR was used in 42 cases and FBP alone in 46. Patients demographic data presented in Table [1] shows no significant differences with respect to sex, age, BMI $(p>0.05)$ between the two employed routes.

\subsection{Image quality}

Spiral CT chest examination SNR and CNR values as listed in Table (3). The average image quality in FBP was superior to that of ASIR images. For BMI less than $30 \mathrm{Kg} / \mathrm{m}^{2}$ SNR were $4.03 \pm 1.79$, $3.00 \pm 1.47$ for FBP, ASIR respectively and there is no significant variation $(p=0.151)$, and CNR were $2.03 \pm 0.61$, $1.17 \pm 0.61$ for $F B P, A S I R$ respectively and there is significant variation $(P=0.003)$.

For BMI more than $30 \mathrm{Kg} / \mathrm{m}^{2}$ SNR $3.33 \pm 1.33,2.12 \pm 0.94$ for FBP, ASIR respectively and there is a significant variation $(p=0.028)$, and CNR were $1.44 \pm 1.01,1.11 \pm 0.53$ for FBP, ASIR respectively and there is nonsignificant variation $(P=0.364)$. 
Table 3. A comparison between CNR and SNR ratios using both FBP and FBP-ASIR routine

\begin{tabular}{|c|c|c|c|c|c|}
\hline \multirow{2}{*}{ BMI } & $\begin{array}{c}\text { CT } \\
\text { parameters }\end{array}$ & $\begin{array}{c}\text { FBP } \\
\mathbf{( n = 2 3 )}\end{array}$ & $\begin{array}{c}\text { ASIR } \\
(\mathbf{n = 2 1 )}\end{array}$ & t-test & p-value \\
\hline \multirow{2}{*}{$<30$} & SNR & $4.03 \pm 1.79$ & $3.00 \pm 1.47$ & 1.49 & 0.151 \\
\cline { 2 - 6 } & CNR & $2.03 \pm 0.61$ & $1.17 \pm 0.61$ & 3.35 & $0.003^{*}$ \\
\hline \multirow{2}{*}{$\geq 30$} & SNR & $3.33 \pm 1.33$ & $2.12 \pm 0.94$ & 2.38 & $0.028^{*}$ \\
\cline { 2 - 6 } & CNR & $1.44 \pm 1.01$ & $1.11 \pm 0.53$ & 0.929 & 0.364 \\
\hline
\end{tabular}

A significant increase in both CNR and SNR combined with increasing ASIR percent was noted and recorded in Table 4. SNR for spiral CT chest examination with BMI less than $30 \mathrm{Kg} / \mathrm{m}^{2}$, SNR was $3.00 \pm 1.47,3.86 \pm 1.82,4.56 \pm 2.08$, and $6.19 \pm 2.77$ for reconstructions with $0 \%, 30 \%, 50 \%$ and 80 ASIR respectively $(P=0.007)$ and $C N R$ was $1.17 \pm 0.61,1.49 \pm 0.73,1.72 \pm 0.80$ and $4.27 \pm 1.6677$ for reconstructions with $0 \%, 30 \%, 50 \%$ and 80 ASIR respectively and $\left(P=0.018^{*}\right)$.

For BMI more than $30 \mathrm{Kg} / \mathrm{m}^{2}$ SNR was $2.12 \pm 0.94,2.74 \pm 1.19,3.28 \pm 1.37$ and $4.27 \pm 1.66$ for reconstructions with $0 \%, 30 \%, 50 \%$ and 80 ASIR respectively $(P=0.006)$ and CNR was $1.11 \pm 0.53$, $1.37 \pm 0.56,1.60 \pm 0.61$ and $2.10 \pm 0.84$ for reconstructions with $0 \%, 30 \%, 50 \%$ and 80 ASIR respectively $(P=0.014)$.

Table (4) SNR and CNR for CT chest examination at different percentage ASIR

\begin{tabular}{|c|c|c|c|c|c|c|c|}
\hline BMI & $\begin{array}{c}\mathrm{CT} \\
\text { parameter }\end{array}$ & $\operatorname{ASIR(0)}$ & $\operatorname{ASIR(30)}$ & $\operatorname{ASIR(50)}$ & ASIR 80 & $\begin{array}{c}\text { ANOVA } \\
\text { test }\end{array}$ & p-value \\
\hline \multirow{2}{*}{$<30$} & SNR & $3.00 \pm 1.47$ & $3.86 \pm 1.82$ & $4.56 \pm 2.08$ & $6.19 \pm 2.77$ & 4.60 & $0.007^{\star}$ \\
\hline & CNR & $1.17 \pm 0.61$ & $1.49 \pm 0.73$ & $1.72 \pm 0.80$ & $2.32 \pm 1.09$ & 3.79 & $0.018^{*}$ \\
\hline \multirow{2}{*}{$\geq 30$} & SNR & $2.12 \pm 0.94$ & $2.74 \pm 1.19$ & $3.28 \pm 1.37$ & $4.27 \pm 1.66$ & 4.78 & $0.006^{*}$ \\
\hline & CNR & $1.11 \pm 0.53$ & $1.37 \pm 0.56$ & $1.60 \pm 0.61$ & $2.10 \pm 0.84$ & 4.07 & $0.014^{*}$ \\
\hline
\end{tabular}

An increased percentage of ASIR has associated a linear improvement in SNR and CNR ((Fig. 2, 3). 


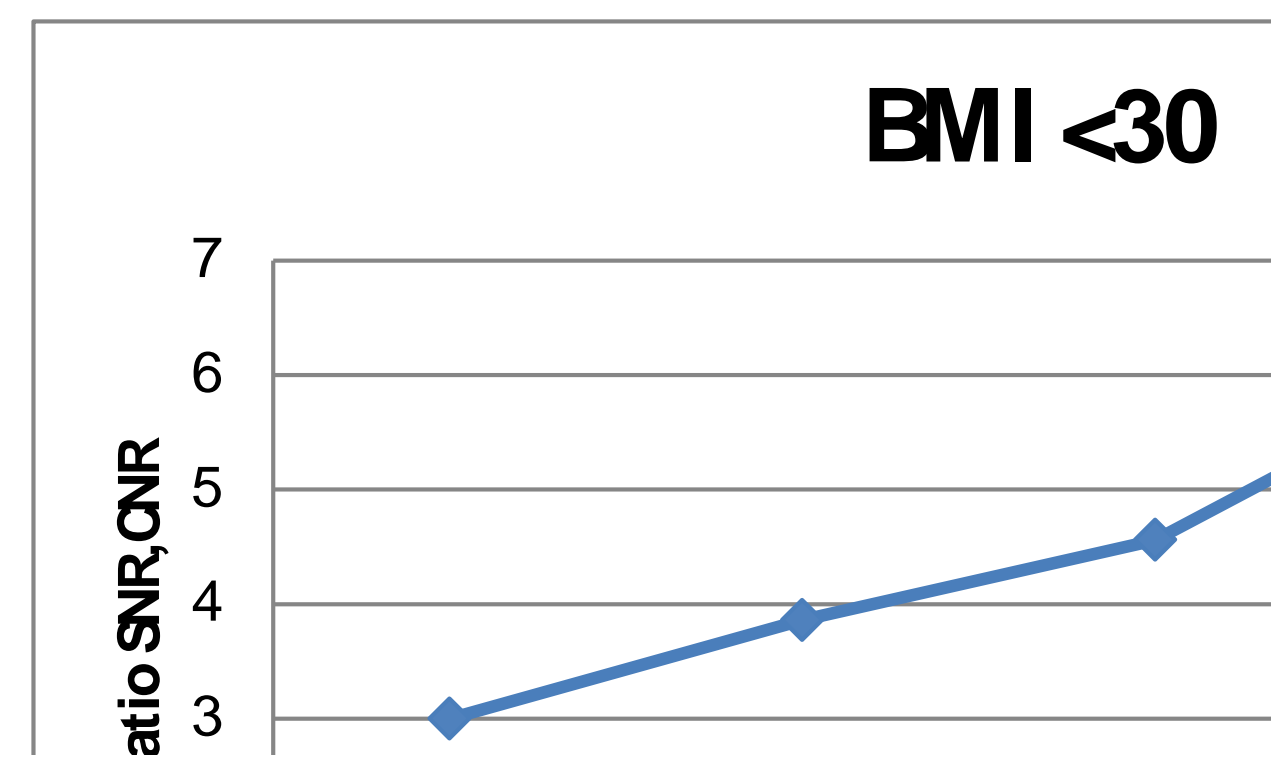

Figure (2) Relation between different ASIR percentages, SNR and CNR in the group of BMI $<30$

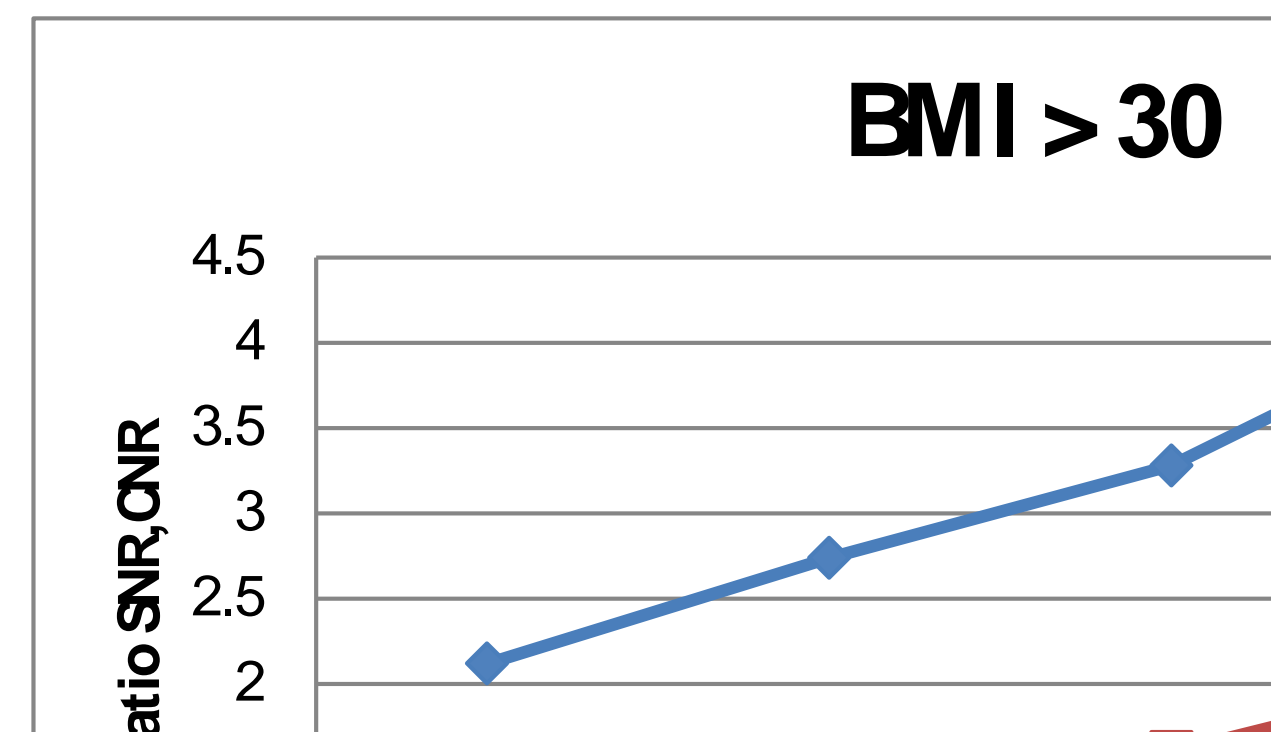

Figure (3) Relation between different ASIR percentages, SNR and CNR in the group of BMI>30 


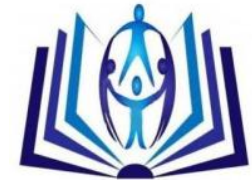

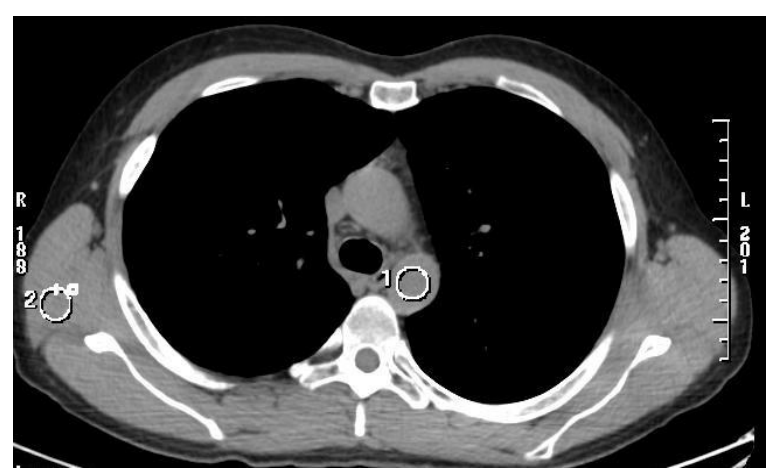

ASIR (0)

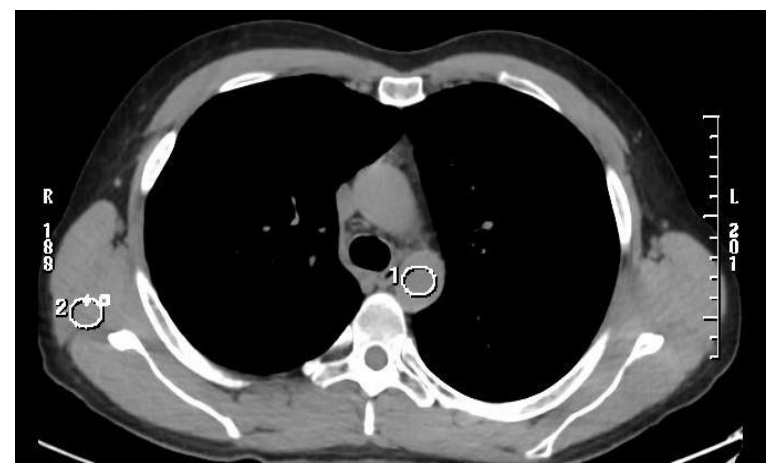

ASIR (50)

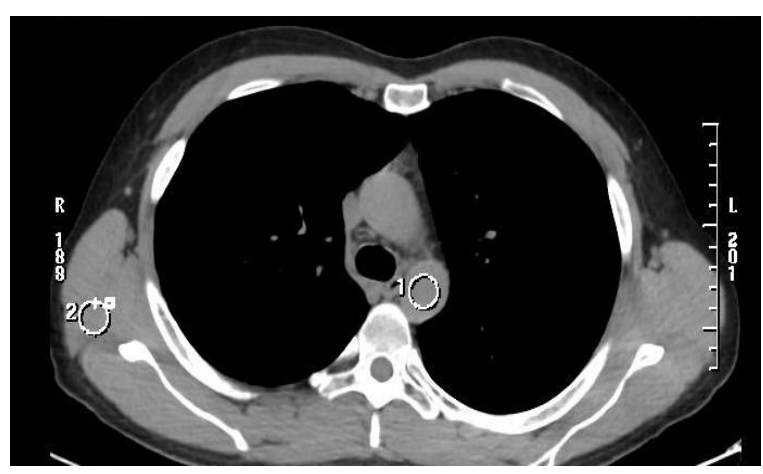

$\operatorname{ASIR}(30)$

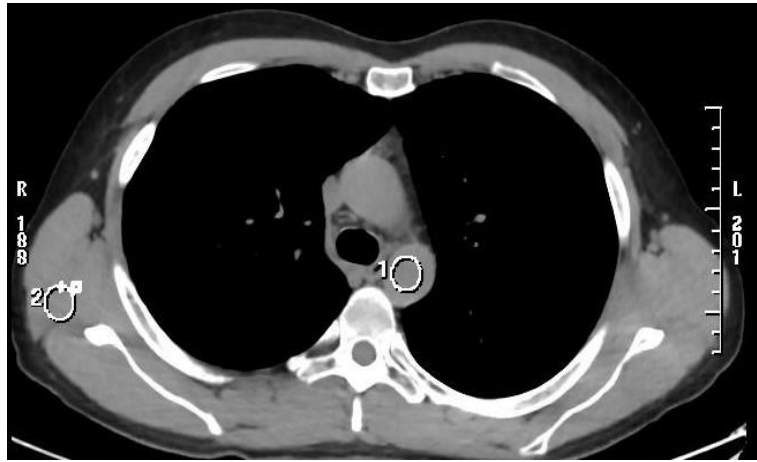

ASIR (80)

Figure (4) A 38 years old male had BMI $24.58 \mathrm{~kg} / \mathrm{cm}^{2}, 2.5 \mathrm{~mm}$ axial non-contrast CT images, Images were reconstructed in ASIR: 0\% (A), 30\% (B), 50\% (C), and $80 \%(D)$.

Figure (4) Image of CT chest examination with increasing percentage of ASIR reconstruction in 38 years old male with BMI (24.58). Images have been reconstructed in ASIR: 0\% (A), 30\% (B), 50\% (C), and $80 \%$ (D). SNR and CNR noted progressive increase with increasing ASIR. SNR was $(2.68,3.41,4.15$ and 5.89), CNR was $(0.97,1.17,1.64$ and 2.22$)$ for reconstructions with: $0 \%(A), 30 \%(B), 50 \%(C)$, and $80 \%$ (D) ASIR respectively.

\subsection{Radiation Dose}

Comparison of CTDIVOL, EDs using FBP and ASIR techniques shown in (Table 5). ASIR had a statistically significantly $(P=0.003)$ lower CTDIvol $(9.02 \pm 2.01)$ than the conventional FBP $(13.52 \pm 4.01)$, For BMI $<30$.

As unexpected for the chest CT examination results reconstructed using ASIR, regardless of $\mathrm{BMI}, \mathrm{ED}$ was slight differ compared with FBP. EDs were range from $7.46 \pm 2.45$, to $9.02 \pm 2.01$ with $\mathrm{BMI}$ $<30 \mathrm{~kg} / \mathrm{m} 2$ ), and were varied from $6.63 \pm 1.59$, to $8.22 \pm 5.13$ with BMI $\geq 30 \mathrm{~kg} / \mathrm{m} 2 \mathrm{for}$ FBP, ASIR respectively. Statistically, there were no significant difference $(P=0.401$ and $P=0.340$ respectively) noted. 
Table (5): CT dose index volume (CTDI vol , effective dose (ED) of FBP and ASIR

\begin{tabular}{|c|c|c|c|c|c|}
\hline \multirow{2}{*}{ BMI } & Doses & FBP & ASIR & t-test & p-value \\
\hline \multirow{2}{*}{$<30$} & CTDI $_{\text {VoL, } m G y}$ & $13.52 \pm 4.01$ & $9.02 \pm 2.01$ & 3.36 & $0.003^{*}$ \\
\cline { 2 - 6 } & $\mathrm{ED}, \mathrm{mSV}$ & $7.46 \pm 2.45$ & $6.59 \pm 2.45$ & 0.857 & 0.401 \\
\hline \multirow{2}{*}{$\geq 30$} & $\mathrm{CTDI}_{\mathrm{VOL}, \mathrm{mGy}}$ & $11.83 \pm 2.86$ & $12.25 \pm 7.49$ & 0.173 & 0.864 \\
\cline { 2 - 6 } & $\mathrm{ED}, \mathrm{mSV}$ & $6.63 \pm 1.59$ & $8.22 \pm 5.13$ & 0.978 & 0.340 \\
\hline
\end{tabular}

Figure $(5,6)$ reveals an inverse relationship between noise index (NI) and radiation dose.

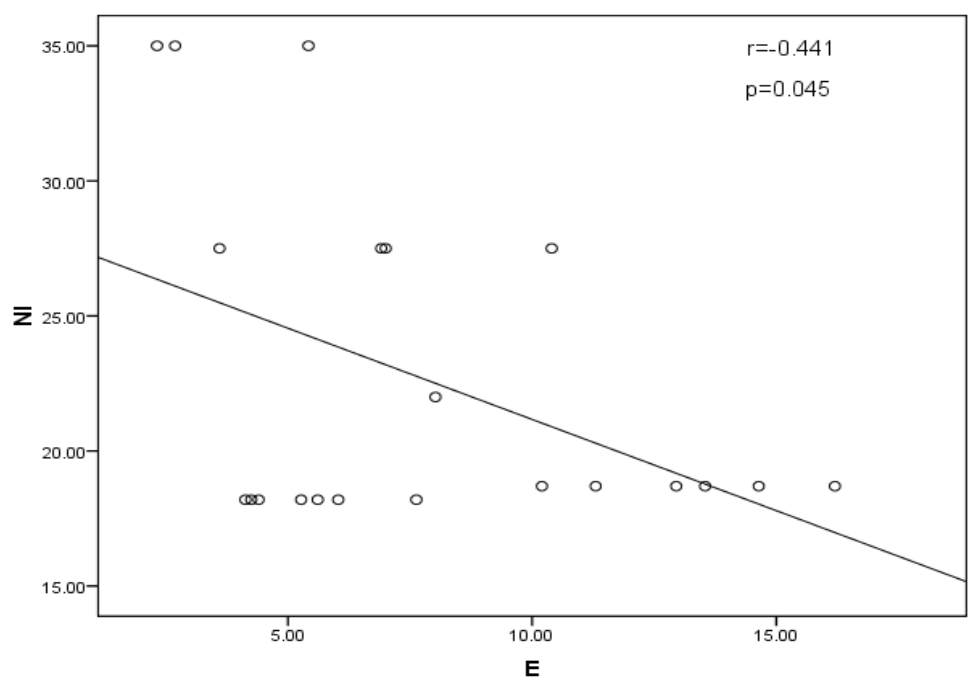

Figure (5): Scatter diagram show correlation between EDs, $\mathrm{mSv}$, and NI by adaptive statistical iterative reconstruction (ASIR), Slopes of the curve were significantly different $(P=0.045)$

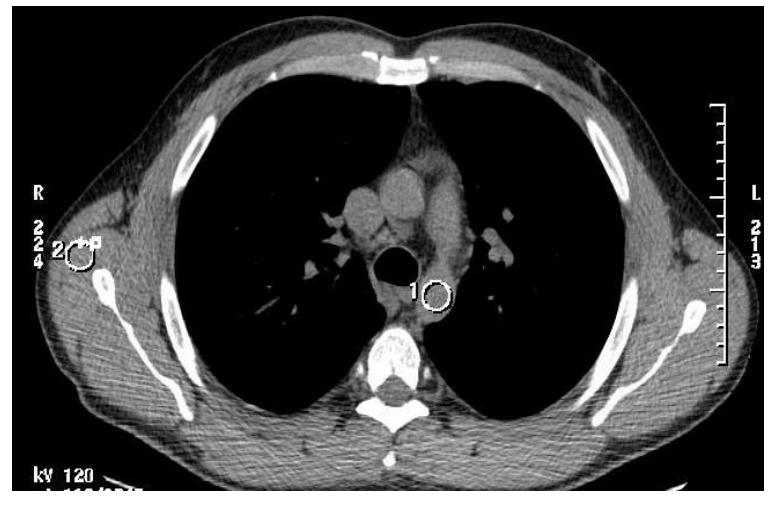

A

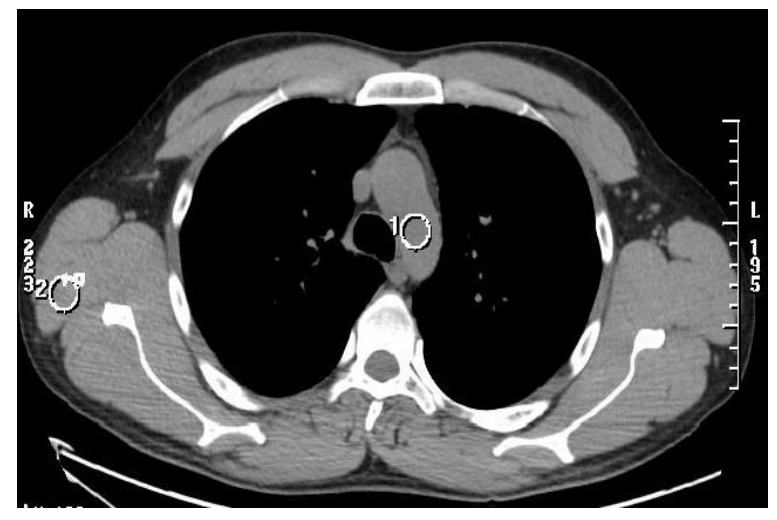

B

Figure (6): Two axial CT chest images for two different patients with the same $\mathrm{BMI}<30$. The $\mathrm{NI}$ and $\mathrm{ED}$ for the image (A) were 35 and 2.69 respectively but for the image (B) were 18.7 and 11.3 respectively. 


\section{Discussion}

The principal essence of the presented study was devoted to identify the clinical effects of ASIR routine on both effective radiation dose and image quality in a non-contrast spiral CT chest examination. 88 were examined by two reconstruction process (FBP and ASIR), we found that the average image quality in FBP was superior to that of ASIR images, SNR were $(4.03 \pm 1.79,3.00 \pm 1.47$ BMI $<30)(3.33 \pm 1.33,2.12 \pm 0.94$, BMI $>30)$, CNR was $(2.03 \pm 0.61,1.17 \pm 0.61, \mathrm{BMI}<30)$, $(1.44 \pm 1.01,1.11 \pm 0.53, \mathrm{BMI}>30)$ for FBP, ASIR respectively. Although, several studies have shown the benefit of ASIR for improving the image quality of CT chest examination [12-14]. Leipsic et al [15] compared CT image quality with FBP and ASIR technique, they found SNR was higher in the ASIR $(4.9 \pm 5.1,6.6 \pm 6.9, p=0.023)$ for FBP, ASIR respectively.

ASIR may use precise statistical modeling methods for image reconstruction using measured signal (noise free) results from x-ray photon and/or electronic noise [16]. This enables increasing noise index, which decreases tube current and radiation dose. According to the study by Pontana et al, there is a direct relationship between the delivered dose and the image noise (when the radiation dose decreases, the image noise increases [17]. In our study, the increase percentage of ASIR resulted in significant noise reduction and improved SNR, $50 \%$ and $80 \%$ ASIR appeared to provide optimal image quality.

Efforts to decrease radiation dose from $\mathrm{CT}$ have focused on developing techniques to reduce radiation dose while maintaining or improving image quality and establishing the clinical value of low-radiation-dose images for diagnostic information [18]. Therefore, CT examination with low-dose may have a much greater noise in formed images which may lead to wrong diagnostic information.

In this study, we found that ASIR had significantly $(P=0.003)$ lower CTDIvol $(9.02 \pm 2.01)$ than the conventional FBP reconstruction (13.52 \pm 4.01$)$. Leipsic et al [15], found that CTDIVOL $(15.4 \pm 6.38$, FBP) and $(11.3 \pm 5$, ASIR) with $\mathrm{P}<0.0001$ and this result agree with our result.

Unfortunately, we did not find any effect in (EDs) by using ASIR technique compared with FBP and there is no dose reduction. Statistically, there were no significant difference $(P=0.401$ and $P=0.340$ respectively) noted. Although, Prakash et al [19] demonstrated that ASIR technique allows a reduction in radiation dose associated with chest $C T$ while reducing image noise, ASIR allows dose reduction by $26 \%$ to $29 \%$ compared with the FBP technique. L-P QI et al showed that radiation doses were significantly lower in the examinations that used ASIR ( $p, 0.001$ ), Dose reduction by $27.7 \%$ to $71.8 \%$ [20]. Additionally, ASIR route supposed to give more noise reduction for the standard low-dose CT chest protocols and it may also results in a more aggressive dose reduction [21].

\section{Conclusion:}

Presented study shows no statistically significant variation in the effective radiation doses related to a non-contrast CT scan of chest reconstructed with FBP or ASIR. The reason was due to the absence of welltrained technicians of the usefulness of the ASIR technology and its ability to maintain the image quality with a low dose. The ASIR reconstruction has not been fully utilized due to the absence of a fixed protocol for all technicians so that individual variation can be reduced. Reconstruction ASIR technique as previous studies can improve image quality with low radiation dose. Further clinical evaluation is required to confirm that ASIR is a considerable method to decrease dose with an acceptable image for the diagnostic of disorders on our institute. For improving the image quality and management radiation dose reduction we recommend that medical physicist should be attended in the radiology department.

\section{Limitations:}

Data obtained from the two sets of patients examined by two scanners using both ASIR and FBP reconstructed routine shows some limitations and there is no significant difference between patients' BMI. The research was not including $C T$ chest with contrast and other $C T$ examination protocol for comparing FBP with ASIR. 


\section{References}

1. National Institute of Environmental Health Sciences Website. List of cancer-causing agents grows.www.niehs.nih.gov/oc/news/canceragents.htm. Accessed March 28, 2007

2. Hall EJ, Brenner DJ. Cancer risks from diagnostic radiology. Br J Radiol 2008; 81:362-378

3. Brenner DJ, Hall EJ. Computed tomography: an increasing source of radiation exposure. $\mathrm{N}$ Engl J Med 2007; 357:2277-2284

4. McNitt-Gray MF. AAPM/RSNA physics tutorial for residents: topics in CT-radiation dose in CT. RadioGraphics 2002; 22:1541-1553

5. Graser A, Wintersperger BJ, Suess $C$, Reiser MF, Becker CR. Dose reduction and image quality in MDCT colonography using tube current modulation. AJR Am J Roentgenol 2006;187:695701

6. Lell $M$, Hinkmann $F$, Anders $K$, Deak $P$, Kalender $W A$, Uder $M$, et al. High-pitch electrocardiogram-triggered computed tomography of the chest. Invest Radiol 2009; 44:72833

7. Campbell J, Kalra MK, Rizzo S, Maher MM, Shepard JA. Scanning beyond anatomic limits of the thorax in chest $\mathrm{CT}$ : findings, radiation dose, and automatic tube current modulation. AJR 2005; 185:1525-1530

8. Campbell J, Kalra MK, Rizzo S, Maher MM, Shepard JA. Scanning beyond anatomic limits of the thorax in chest CT: findings, radiation dose, and automatic tube current modulation. AJR 2005; 185:1525

9. Alvin C. Silva, Holly J. Lawder, Amy Hara, Jennifer Kujak, William Pavlicek. Innovations in CT Dose Reduction Strategy: Application of the Adaptive Statistical Iterative Reconstruction Algorithm. AJR 2010; 194:191-199

10. Szucs-Farkas Z, Strautz T, Patak MA, Kurmann L, Vock P, Schindera ST (2009) Is body weight the most appropriate criterion to select patients eligible for low-dose pulmonary $C T$ angiography? Analysis of objective and subjective image quality at $80 \mathrm{kVp}$ in 100 patients. Eur Radiol 19:1914-1922

11. EUR 16262. Quality criteria for computed tomography. Available at: www.drs.dk/guidelines/ct/quality/download/eur16262.w51.Accessed December 29, 2008

12. Prakash $\mathrm{P}$, Kalra MK, Digumarthy $S R$, Hsieh J, Pien $H$, Singh $S$, et al. Radiation dose reduction with chest computed tomography using adaptive statistical iterative reconstruction technique: initial experience. J Comput Assist Tomogr 2010;34:40-45

13. Prakash P, Kalra MK, Ackman JB, Digumarthy SR, Hsieh J, Do S, et al. Diffuse lung disease: CT of the chest with adaptive statistical iterative reconstruction technique. Radiology 2010;256:261-269

14. Yanagawa $M$, Honda $O$, Yoshida S, Kikuyama A, Inoue $A$, Sumikawa $H$, et al. Adaptive statistical iterative reconstruction technique for pulmonary $\mathrm{CT}$ : image quality of the cadaveric lung on standard- and reduced-dose CT. Acad Radiol 2010;17:1259-1266

15. Jonathon Leipsic, Giang Nguyen, Jaqueline Brown, Don Sin, John R. Mayo. A Prospective Evaluation of Dose Reduction and Image Quality in Chest CT Using Adaptive Statistical Iterative Reconstruction. AJR 2010; 195:1095-1099. 
16. Cheng LCY, Fang T, Tyan J. Fast iterative adaptive reconstruction in low-dose CT imaging. Proceedings of the IEEE International Conference on Image Processing. New York, NY: IEEE. 2006:889-892

17. Pontana F, Duhamel A, Pagniez J et al (2011) Chest computed tomography using iterative reconstruction vs. filtered back projection (part 2): image quality of low-dose CT examinations in 80 patients. Eur Radiol 21:636-643

18. Kalra MK, Maher MM, Toth $\mathrm{TL}$, et al. Strategies for $\mathrm{CT}$ radiation dose optimization. Radiology. 2004;230:619Y628.

19. Priyanka Prakash, Mannudeep K. Kalra, Subba R. Digumarthy, Jiang Hsieh, Homer Pien. Radiation Dose Reduction With Chest Computed Tomography Using Adaptive Statistical Iterative Reconstruction Technique: Initial Experience. J Comput Assist Tomogr 2010;34: 40Y45

20. L-P QI, Y LI, L TANG, Y-L LI, X-T LI. Evaluation of dose reduction and image quality in chest CT using adaptive statistical iterative reconstruction with the same group of patients. The British Journal of Radiology, 85 (2012), e906-e911.

21. Alvin C. Silva, Holly J. Lawder, Amy Hara, Jennifer Kujak, William Pavlicek.Innovations in CT Dose Reduction Strategy: Application of the Adaptive Statistical Iterative Reconstruction Algorithm. AJR 2010; 194:191-199 